\title{
COMPACT DIFFERENCES OF COMPOSITION OPERATORS ON BERGMAN SPACES IN THE BALL
}

\author{
XIANG DONG YANG ${ }^{\otimes}$ and LE HAI KHOI
}

(Received 27 July 2009; accepted 3 January 2011)

Communicated by A. M. Hassell

\begin{abstract}
We obtain necessary and sufficient conditions for the compactness of differences of composition operators acting on the weighted Bergman spaces in the unit ball. A representation of a composition operator as a finite sum of composition operators modulo compact operators is also studied.
\end{abstract}

2010 Mathematics subject classification: primary 32A36; secondary 47B33.

Keywords and phrases: Bergman space, composition operator, compact difference.

\section{Introduction}

1.1. Notation and definitions. We begin with some basic notation and definitions. For $z, \zeta \in \mathbb{C}^{n}$, we set $\langle z, \zeta\rangle=z_{1} \bar{\zeta}_{1}+\cdots+z_{n} \bar{\zeta}_{n}$ and $|z|=\left(z_{1} \bar{z}_{1}+\cdots+z_{n} \bar{z}_{n}\right)^{1 / 2}$.

Let $\mathbb{B}$ denote the unit ball in $\mathbb{C}^{n}$, and $\mathcal{O}(\mathbb{B})$ denote the space of holomorphic functions on $\mathbb{B}$, with the topology of uniform convergence on compact subsets of $\mathbb{B}$. Let $V$ denote the normalized Lebesgue measure on $\mathbb{B}$, that is, $V(\mathbb{B})=1$. For all $\alpha>-1$, define the measure $V_{\alpha}$ by

$$
d V_{\alpha}(z)=c_{\alpha}\left(1-|z|^{2}\right)^{\alpha} d V(z)
$$

where $c_{\alpha}$ is a positive normalizing constant, that is, $V_{\alpha}(\mathbb{B})=1$. When $p>0$ and $\alpha>-1$, denote by $A_{\alpha}^{p}(\mathbb{B})$, or just $A_{\alpha}^{p}$, the weighted Bergman space in $\mathbb{B}$ :

$$
A_{\alpha}^{p}(\mathbb{B}):=\left\{f \in \mathcal{O}(\mathbb{B}): \int_{\mathbb{B}}|f(z)|^{p} d V_{\alpha}(z)<\infty\right\} .
$$

The first author is supported by the Natural Science Foundation of Yunnan Province in China (Grant No. 2009ZC013X) and the Basic Research Foundation of the Education Bureau of Yunnan Province in China (Grant No. 09Y0079).

(C) 2011 Australian Mathematical Publishing Association Inc. 1446-7887/2011 \$16.00 
When $p=2$, the space $A_{\alpha}^{2}$ is a Hilbert space, with inner product given by

$$
\langle f, g\rangle=\int_{\mathbb{B}} f(z) \overline{g(z)} d V_{\alpha}(z) .
$$

If $n=1, p=2$, and $\alpha>-1$, then, taking $c_{\alpha}=(\alpha+1) / \pi$, we have the standard weighted Bergman space $A_{\alpha}^{2}(\mathbb{D})$ on the unit disk $\mathbb{D}$ in the complex plane $\mathbb{C}$.

1.2. Composition operators. Each holomorphic mapping $\varphi: \mathbb{B} \rightarrow \mathbb{B}$ determines a linear composition operator $C_{\varphi}: \mathcal{O}(\mathbb{B}) \rightarrow \mathcal{O}(\mathbb{B})$ by

$$
C_{\varphi}(f)(z)=f \circ \varphi(z) \quad \forall z \in \mathbb{B} .
$$

Composition operators acting on spaces of analytic functions in the unit disk have been studied in some detail and many phenomena are well understood. We refer the reader to the monographs [1,9] for more information. Much effort has been spent on understanding the compactness of composition operators on $A_{\alpha}^{2}(\mathbb{D})$, and on the compactness of differences of composition operators. In particular, motivated by [10], the following results were obtained in [6] (see also [7]).

TheOREM 1.1. Suppose that $\alpha>-1$. Let $\varphi, \psi: \mathbb{D} \rightarrow \mathbb{D}$ be holomorphic mappings, and write $\rho(z)$ for the pseudohyperbolic distance $|\varphi(z)-\psi(z)| /|1-\bar{\varphi}(z) \psi(z)|$ between $\varphi(z)$ and $\psi(z)$. Then the following assertions are equivalent.

(a) $C_{\varphi}-C_{\psi}$ is compact on $A_{\alpha}^{2}(\mathbb{D})$.

(b) $\lim _{|z| \rightarrow 1} \rho(z)\left(\left(1-|z|^{2}\right) /\left(1-|\varphi(z)|^{2}\right)+\left(1-|z|^{2}\right) /\left(1-|\psi(z)|^{2}\right)\right)=0$.

The notation $A \equiv B \bmod K$ indicates that the difference $K$ between two bounded operators $A$ and $B$ is compact.

TheOREM 1.2. Suppose that $\alpha>-1$. Let $\varphi, \varphi_{1}, \ldots, \varphi_{N}: \mathbb{D} \rightarrow \mathbb{D}$ be holomorphic mappings. Let $F$ and $F_{i}$ (where $i=1, \ldots, N$ ) be the set of points on the boundary of the disk $\mathbb{D}$ at which $\varphi$ and $\varphi_{i}$ have finite angular derivatives. Suppose that $F_{i} \cap F_{j}=\varnothing$ whenever $i \neq j$, and $F=\bigcup_{i=1}^{N} F_{i}$. Define $\rho_{i}(z)=\left|\varphi(z)-\varphi_{i}(z)\right| /\left|1-\bar{\varphi}(z) \varphi_{i}(z)\right|$ (where $i=1, \ldots, N)$. If

$$
\lim _{z \rightarrow \zeta} \rho_{i}(z)\left(\frac{1-|z|^{2}}{1-\left|\varphi_{i}(z)\right|^{2}}+\frac{1-|z|^{2}}{1-|\varphi(z)|^{2}}\right)=0
$$

for every $\zeta \in F_{i}$ when $i=1, \ldots, N$, then there exists a compact operator $K$ on $A_{\alpha}^{2}(\mathbb{D})$ such that

$$
C_{\varphi} \equiv C_{\varphi_{1}}+\cdots+C_{\varphi_{N}} \bmod K
$$

We may ask the question: what is the picture in higher dimensions?

Although composition operators in several variables are usually harder to deal with, there is some active research going on. We flag the papers [2-4, 12], which contain key references for recent developments in this direction. 
The goal of this paper is to generalize Theorems 1.1 and 1.2 to the case of the ball in $\mathbb{C}^{n}$, and more precisely, to the weighted Bergman spaces $A_{\alpha}^{p}(\mathbb{B})$. It should be noted that the proofs of Theorems 1.1 and 1.2 in $[6,7]$, based on the results from [1], do not seem to apply in the situation of a higher dimension. Our method uses Schur's test; this seems to be new in the study of compact differences.

1.3. Main results. First we need some basic facts.

As is well known (see, for instance, [8, pp. 25-27]), given a point $a \in \mathbb{B}$, we may associate to it the following automorphism:

$$
\Phi_{a}(z)=\frac{a-P_{a} z-s_{a} Q_{a} z}{1-\langle z, a\rangle} \quad \forall z \in \mathbb{B},
$$

where $s_{a}=\left(1-|a|^{2}\right)^{1 / 2}$ and $P_{a}$ is the orthogonal projection of $\mathbb{C}^{n}$ onto the subspace $\mathbb{C} a$ generated by $a$, that is,

$$
P_{a} z= \begin{cases}0 & \text { if } a=0, \\ \frac{\langle z, a\rangle}{\langle a, a\rangle} a & \text { if } a \neq 0,\end{cases}
$$

and $Q_{a}=I-P_{a}$, the projection onto the orthogonal complement of $\mathbb{C} a$. The automorphism $\Phi_{a}(z)$ has the following important property:

$$
1-\left|\Phi_{a}(z)\right|^{2}=\frac{\left(1-|a|^{2}\right)\left(1-|z|^{2}\right)}{|1-\langle z, a\rangle|^{2}} \quad \forall z \in \overline{\mathbb{B}} .
$$

For two holomorphic mappings $\varphi, \psi: \mathbb{B} \rightarrow \mathbb{B}$, define $\rho_{\varphi, \psi}$ by

$$
\rho_{\varphi, \psi}(z)=\left|\Phi_{\varphi(z)}(\psi(z))\right| .
$$

Then (1.2) becomes

$$
1-\left|\rho_{\varphi, \psi}(z)\right|^{2}=\frac{\left(1-|\varphi(z)|^{2}\right)\left(1-|\psi(z)|^{2}\right)}{|1-\langle\varphi(z), \psi(z)\rangle|^{2}} \quad \forall z \in \mathbb{B} .
$$

Evidently $\rho_{\varphi, \psi}=\rho_{\psi, \varphi}$. We are now ready to formulate the main results of this paper.

THEOREM 1.3. Suppose that $p>0, q>0$ and $\alpha>\beta>-1$. Let $\varphi, \psi$ be holomorphic self-mappings on $\mathbb{B}$. Suppose that $C_{\varphi}$ and $C_{\psi}$ are bounded on $A_{\beta}^{q}$. The difference of composition operators $C_{\varphi}-C_{\psi}$ is compact on $A_{\alpha}^{p}$ if and only if

$$
\lim _{|z| \rightarrow 1} \rho_{\varphi, \psi}(z)\left(\frac{1-|z|^{2}}{1-|\varphi(z)|^{2}}+\frac{1-|z|^{2}}{1-|\psi(z)|^{2}}\right)=0 .
$$

THEOREM 1.4. Suppose that $p>0, q>0$ and $\alpha>\beta>-1$. Let $\varphi_{0}, \varphi_{1}, \ldots, \varphi_{N}$ be holomorphic self-mappings on $\mathbb{B}$. Suppose that $C_{\varphi_{0}}, C_{\varphi_{1}}, \ldots, C_{\varphi_{N}}$ are bounded on $A_{\beta}^{q}$. If

$$
\lim _{|z| \rightarrow 1} \rho_{\varphi_{i}, \varphi_{j}}(z)\left(\frac{1-|z|^{2}}{1-\left|\varphi_{i}(z)\right|^{2}}+\frac{1-|z|^{2}}{1-\left|\varphi_{j}(z)\right|^{2}}\right)=0
$$


when $0 \leq i, j \leq n$, then there exists a compact operator $K$ on $A_{\alpha}^{p}$ such that

$$
C_{\varphi} \equiv C_{\varphi_{1}}+\cdots+C_{\varphi_{N}} \bmod K
$$

REMARK 1.5. It is well-known that, when $n=1$, composition operators are always bounded on $A_{\alpha}^{p}$. In this case, the assumption on boundedness in Theorems 1.3 and 1.4 is automatically satisfied.

\section{Proofs of the theorems}

As Schur's test plays a crucial role in our proof, we present it here for the reader's convenience. This version of Schur's test is concerned with the boundedness of integral operators on $L^{2}$ spaces. It may be found, for example, in [5]; see also [13, pp. 52-54].

LEMMA 2.1. Let $(X, \mu)$ and $(Y, v)$ be measure spaces, and let $T$ be the integral operator with nonnegative kernel $K: X \times Y \rightarrow[0, \infty)$, defined by

$$
(T f)(x)=\int_{Y} K(x, y) f(y) d v(y)
$$

(for almost all $x$ in $X$ ). Suppose that there exist positive measurable functions $h$ on $X$ and $g$ on $Y$ and numbers $A$ and $B$ such that

$$
\int_{X} K(x, y) h(x) d \mu(x) \leq A g(y)
$$

for almost all $y$ in $Y$, and

$$
\int_{Y} K(x, y) g(y) d \nu(y) \leq B h(x)
$$

for almost all $x$ in $X$. Then $T$ is bounded from $L^{2}(X, \mu)$ to $L^{2}(Y, v)$, and its operator norm $\|T\|$ is at most $\sqrt{A B}$.

The proof of this test is quite simple; it uses uses only the Cauchy-Schwarz inequality and Fubini's theorem.

2.1. Proof of Theorem 1.3. We know (see, for instance, [14] or [1, p. 164]) that the boundedness and compactness of composition operators on $A_{\alpha}^{p}$ does not depend on $p$. More precisely, if $p>0, q>0$ and $\alpha>-1$, then the operator $C_{\varphi}$ is bounded or compact on $A_{\alpha}^{p}$ if and only if $C_{\varphi}$ is bounded or compact on $A_{\alpha}^{q}$. Therefore, we just need to prove the theorem when $p=2$.

Let $C_{\varphi}$ and $C_{\psi}$ be bounded on $A_{\beta}^{q}$ for some $q>0$ and $\beta \in(-1, \alpha)$. In this case, $\sigma=\alpha-\beta>0$. As we will see below, the weighted Bergman space $A_{\beta}^{\sigma}$ plays an important role in our discussion.

Necessity. Suppose that (1.5) holds. We must show that $C_{\varphi}-C_{\psi}$ is compact on $A_{\alpha}^{2}$. 
By [14], the operator

$$
C_{\varphi}^{*} C_{\varphi}: A_{\alpha}^{2} \rightarrow A_{\alpha}^{2}
$$

where $C_{\varphi}^{*}$ is the adjoint to $C_{\varphi}$, admits the integral representation

$$
C_{\varphi}^{*} C_{\varphi} f(z)=\int_{\mathbb{B}} \frac{f(w)}{(1-\langle\varphi(z), \varphi(w)\rangle)^{n+1+\alpha}} d V_{\alpha}(w) \quad \forall f \in A_{\alpha}^{2} \forall z \in \mathbb{B} .
$$

For all $\gamma \in(0,1)$, define the set $S_{\gamma}$ by

$$
S_{\gamma}=\left\{z \in \mathbb{B}: \gamma<\left[\rho_{\varphi, \psi}(z)\right]^{\sigma}<1\right\},
$$

and let $\chi_{\gamma}$ be its characteristic function, where $\rho_{\varphi, \psi}$ is defined by (1.3). Consider the integral operator $T$ on $L^{2}\left(\mathbb{B}, V_{\alpha}\right)$ given by

$$
\left(T_{\gamma} f\right)(z)=\int_{\mathbb{B}} K_{\gamma}(z, w) f(w) d V_{\alpha}(w) \quad \forall f \in L^{2}(\mathbb{B}) \forall z \in \mathbb{B},
$$

where the nonnegative integral kernel $K_{\gamma}$ is given by

$$
\begin{aligned}
K_{\gamma}(z, w)= & \frac{\left[\rho_{\varphi, \psi}(z)\right]^{\sigma}\left[\rho_{\varphi, \psi}(w)\right]^{\sigma} \chi_{\gamma}(z) \chi_{\gamma}(w)}{|1-\langle\varphi(z), \varphi(w)\rangle|^{n+1+\alpha}} \\
& +\frac{\left[\rho_{\varphi, \psi}(z)\right]^{\sigma}\left[\rho_{\varphi, \psi}(w)\right]^{\sigma} \chi_{\gamma}(z) \chi_{\gamma}(w)}{|1-\langle\psi(z), \psi(w)\rangle|^{n+1+\alpha}} \\
= & K_{\varphi, \gamma}(z, w)+K_{\psi, \gamma}(z, w),
\end{aligned}
$$

say, for all $w, z \in \mathbb{B}$.

Define $h(z)=\left(1-|z|^{2}\right)^{-\sigma}$ for all $z \in \mathbb{B}$. Taking into account the definition of the normalizing constants $c_{\alpha}$ and $c_{\beta}$, we see that

$$
\begin{aligned}
\int_{\mathbb{B}} K_{\varphi, \gamma}(z, w) h(w) d V_{\alpha}(w)= & \frac{c_{\alpha}}{c_{\beta}} \int_{\mathbb{B}} \frac{\left[\rho_{\varphi, \psi}(z)\right]^{\sigma}\left[\rho_{\varphi, \psi}(w)\right]^{\sigma} \chi_{\gamma}(z) \chi_{\gamma}(w)}{|1-\langle\varphi(z), \varphi(w)\rangle|^{n+1+\alpha}} d V_{\beta}(w) \\
\leq & \frac{c_{\alpha}}{c_{\beta}} \int_{\mathbb{B}} \frac{\left[\rho_{\varphi, \psi}(z)\right]^{\sigma} \chi_{\gamma}(z)}{|1-\langle\varphi(z), \varphi(w)\rangle|^{n+1+\alpha}} d V_{\beta}(w) \\
= & \frac{c_{\alpha}}{c_{\beta}}\left[\rho_{\varphi, \psi}(z)\right]^{\sigma} \chi_{\gamma}(z) \\
& \times \int_{\mathbb{B}} \frac{1}{|1-\langle\varphi(z), \varphi(w)\rangle|^{n+1+\alpha}} d V_{\beta}(w) .
\end{aligned}
$$

By the boundedness of $C_{\varphi}$ on $A_{\beta}^{q}$, there exists a constant $c_{1}>0$, independent of $\gamma$ and $z$, such that

$$
\int_{\mathbb{B}} \frac{1}{|1-\langle\varphi(z), \varphi(w)\rangle|^{n+1+\alpha}} d V_{\beta}(w) \leq c_{1} \int_{\mathbb{B}} \frac{1}{|1-\langle\varphi(z), w\rangle|^{n+1+\alpha}} d V_{\beta}(w),
$$


and hence

$\int_{\mathbb{B}} K_{\varphi, \gamma}(z, w) h(w) d V_{\alpha}(w) \leq c_{2}\left[\rho_{\varphi, \psi}(z)\right]^{\sigma} \chi_{\gamma}(z) \int_{\mathbb{B}} \frac{1}{|1-\langle\varphi(z), w\rangle|^{n+1+\alpha}} d V_{\beta}(w)$,

where $c_{2}=c_{1} c_{\alpha} / c_{\beta}$.

Define

$$
\begin{aligned}
M_{\rho, \gamma} & =\sup _{z \in S_{\gamma}} \rho_{\varphi, \psi}(z) \frac{1-|z|^{2}}{1-|\varphi(z)|^{2}}+\sup _{z \in S_{\gamma}} \rho_{\varphi, \psi}(z) \frac{1-|z|^{2}}{1-|\psi(z)|^{2}} \\
& =M_{\varphi}\{\rho, \gamma\}+M_{\psi}\{\rho, \gamma\}
\end{aligned}
$$

say. Since $\beta>-1$ and $\sigma>0$, by [8, Ch. 2, Section 2], there exists a positive constant $c_{3}$, independent of $\gamma$ and $z$, such that

$$
\begin{aligned}
\int_{\mathbb{B}} \frac{1}{|1-\langle\varphi(z), w\rangle|^{n+1+\alpha}} d V_{\beta}(w) & =\int_{\mathbb{B}} \frac{1}{|1-\langle\varphi(z), w\rangle|^{n+1+\beta+\sigma}} d V_{\beta}(w) \\
& \leq \frac{c_{3}}{\left(1-|\varphi(z)|^{2}\right)^{\sigma}} \quad \forall z \in \mathbb{B}
\end{aligned}
$$

Thus we obtain

$$
\begin{aligned}
\int_{\mathbb{B}} K_{\varphi, \gamma}(z, w) h(w) d V_{\alpha}(w) & \leq \frac{c_{2} c_{3}\left[\rho_{\varphi, \psi}(z)\right]^{\sigma} \chi_{\gamma}(z)}{\left(1-|\varphi(z)|^{2}\right)^{\sigma}} \\
& =c_{4} \chi_{\gamma}(z)\left(\rho_{\varphi, \psi}(z) \frac{1-|z|^{2}}{1-|\varphi(z)|^{2}}\right)^{\sigma} h(z) \\
& \leq c_{4}\left[M_{\varphi}\{\rho, \gamma\}\right]^{\sigma} h(z)
\end{aligned}
$$

for all $z \in \mathbb{B}$, where $c_{4}=c_{2} c_{3}$.

By the symmetry of $K_{\varphi, \gamma}$,

$$
\int_{\mathbb{B}} K_{\varphi, \gamma}(z, w) h(z) d V_{\alpha}(z) \leq c_{4}\left[M_{\varphi}\{\rho, \gamma\}\right]^{\sigma} h(w) \quad \forall w \in \mathbb{B} .
$$

Replacing $\varphi$ in (2.2) by $\psi$, we see similarly that the same conclusion holds for the kernel $K_{\psi, \gamma}$ determined by $\psi$, that is, there is a positive constant $c_{4}^{\prime}$ such that

$$
\int_{\mathbb{B}} K_{\psi, \gamma}(z, w) h(z) d V_{\alpha}(z) \leq c_{4}^{\prime}\left[M_{\psi}\{\rho, \gamma\}\right]^{\sigma} h(w) \quad \forall w \in \mathbb{B} .
$$

Applying Schur's test as in Lemma 2.1 to the operator $T_{\gamma}$ defined by (2.4), we see that $T_{\gamma}$ is bounded on $L^{2}\left(\mathbb{B}, V_{\alpha}\right)$, and its operator norm on this space satisfies

$$
\|T\| \leq c_{5}\left[M_{\rho, \gamma}\right]^{\sigma}
$$

where the constant $c_{5}$ is at $\operatorname{most} \max \left\{c_{4}, c_{4}^{\prime}\right\}$. 
Now fix $\gamma \in[1 / 2,1)$ and a bounded sequence $\left(f_{k}\right)$ in $A_{\alpha}^{2}$ that converges to 0 uniformly on every compact subset of $\mathbb{B}$. We will show that

$$
\left\|\left(C_{\varphi}-C_{\psi}\right) f_{k}\right\|^{2} \rightarrow 0 .
$$

By the integral representation (2.2),

$$
\left(C_{\varphi}-C_{\psi}\right)^{*}\left(C_{\varphi}-C_{\psi}\right) f_{k}=R_{k}+G_{k}
$$

say, where

$$
\begin{aligned}
R_{k}(z)= & \int_{\mathbb{B} \backslash S_{\gamma}}\left(\frac{f_{k}(w)}{(1-\langle\varphi(z), \varphi(w)\rangle)^{n+1+\alpha}}-\frac{f_{k}(w)}{(1-\langle\psi(z), \varphi(w)\rangle)^{n+1+\alpha}}\right. \\
& \left.-\frac{f_{k}(w)}{(1-\langle\varphi(z), \psi(w)\rangle)^{n+1+\alpha}}+\frac{f_{k}(w)}{(1-\langle\psi(z), \psi(w)\rangle)^{n+1+\alpha}}\right) d V_{\alpha}(w)
\end{aligned}
$$

and

$$
\begin{aligned}
G_{k}(z)=\int_{S_{\gamma}} & \left(\frac{f_{k}(w)}{(1-\langle\varphi(z), \varphi(w)\rangle)^{n+1+\alpha}}-\frac{f_{k}(w)}{(1-\langle\psi(z), \varphi(w)\rangle)^{n+1+\alpha}}\right. \\
& \left.-\frac{f_{k}(w)}{(1-\langle\varphi(z), \psi(w)\rangle)^{n+1+\alpha}}+\frac{f_{k}(w)}{(1-\langle\psi(z), \psi(w)\rangle)^{n+1+\alpha}}\right) d V_{\alpha}(w)
\end{aligned}
$$

the set $S_{\gamma}$ is given by (2.3). On one hand, since the sequence $\left(f_{k}(w)\right)$ converges to 0 , uniformly for $w$ in $\mathbb{B} \backslash S_{\gamma}$,

$$
\lim _{k \rightarrow \infty} \int_{\mathbb{B}}\left|R_{k}(z)\right|^{2} d V_{\alpha}(z)=0 .
$$

On the other hand, we may write $G_{k}(z)$ as

$$
\begin{aligned}
\int_{\mathbb{B}} & \left(\frac{\chi_{\gamma}(w) f_{k}(w)}{(1-\langle\varphi(z), \varphi(w)\rangle)^{n+1+\alpha}}-\frac{\chi_{\gamma}(w) f_{k}(w)}{(1-\langle\psi(z), \varphi(w)\rangle)^{n+1+\alpha}}\right. \\
- & \left.\frac{\chi_{\gamma}(w) f_{k}(w)}{(1-\langle\varphi(z), \psi(w)\rangle)^{n+1+\alpha}}+\frac{\chi_{\gamma}(w) f_{k}(w)}{(1-\langle\psi(z), \psi(w)\rangle)^{n+1+\alpha}}\right) d V_{\alpha}(w) .
\end{aligned}
$$

By the weak convergence of $\left(f_{k}\right)$ to 0 in $L^{2}\left(\mathbb{B}, V_{\alpha}\right)$,

$$
\lim _{k \rightarrow \infty} G_{k}(z)=0 \quad \forall z \in \mathbb{B} .
$$

Furthermore, we may also write $G_{k}(z)$ as

$$
\begin{gathered}
\int_{\mathbb{B}}\left(\frac{\chi_{\gamma}(w) f_{k}(w)}{(1-\langle\varphi(z), \varphi(w)\rangle)^{n+1+\alpha}}-\frac{\chi_{\gamma}(w) f_{k}(w)}{(1-\langle\psi(z), \varphi(w)\rangle)^{n+1+\alpha}}\right. \\
\left.-\frac{\chi_{\gamma}(w) f_{k}(w)}{(1-\langle\varphi(z), \psi(w)\rangle)^{n+1+\alpha}}+\frac{\chi_{\gamma}(w) f_{k}(w)}{(1-\langle\psi(z), \psi(w)\rangle)^{n+1+\alpha}}\right)
\end{gathered}
$$




$$
\begin{aligned}
& \times\left(1-\left[\rho_{\varphi, \psi}(w)\right]^{\sigma}\right) d V_{\alpha}(w)+\int_{\mathbb{B}}\left(\frac{\chi_{\gamma}(w) f_{k}(w)}{(1-\langle\varphi(z), \varphi(w)\rangle)^{n+1+\alpha}}\right. \\
& -\frac{\chi_{\gamma}(w) f_{k}(w)}{(1-\langle\psi(z), \varphi(w)\rangle)^{n+1+\alpha}}-\frac{\chi_{\gamma}(w) f_{k}(w)}{(1-\langle\varphi(z), \psi(w)\rangle)^{n+1+\alpha}} \\
& \left.+\frac{\chi_{\gamma}(w) f_{k}(w)}{(1-\langle\psi(z), \psi(w)\rangle)^{n+1+\alpha}}\right)\left[\rho_{\varphi, \psi}(w)\right]^{\sigma} d V_{\alpha}(w),
\end{aligned}
$$

from which, as $\gamma \geq 1 / 2$, it follows that

$$
\left|G_{k}(z)\right| \leq 4 \int_{\mathbb{B}}\left(\frac{\chi_{\gamma}(w)\left[\rho_{\varphi, \psi}(w)\right]^{\sigma}\left|f_{k}(w)\right|}{|1-\langle\varphi(z), \varphi(w)\rangle|^{n+1+\alpha}}+\frac{\chi_{\gamma}(w)\left[\rho_{\varphi, \psi}(w)\right]^{\sigma}\left|f_{k}(w)\right|}{|1-\langle\psi(z), \psi(w)\rangle|^{n+1+\alpha}}\right) d V_{\alpha}(w) .
$$

Moreover,

$$
\int_{\mathbb{B}}\left|G_{k}(z)\right|^{2} d V_{\alpha}(z) \leq \int_{\mathbb{B} \backslash S_{\gamma^{1 / 2}}}\left|G_{k}(z)\right|^{2} d V_{\alpha}(z)+\int_{S_{\gamma^{1 / 2}}}\left|G_{k}(z)\right|^{2} d V_{\alpha}(z),
$$

and therefore

$$
\int_{\mathbb{B}}\left|G_{k}(z)\right|^{2} d V_{\alpha}(z) \leq \int_{\mathbb{B} \backslash S_{\gamma^{1 / 2}}}\left|G_{k}(z)\right|^{2} d V_{\alpha}(z)+c_{5} \int_{\mathbb{B}}\left|T_{\gamma}\left(\left|f_{k}\right|\right)\right|^{2} d V_{\alpha}(z),
$$

for some positive constant $c_{5}$, by the same arguments as for (2.10) applied to the measure $V_{\alpha}$.

From the estimate (2.5) on the norm of $T_{\gamma}$ above, we see that there exists a positive constant $c_{6}$ such that

$$
\int_{\mathbb{B}}\left|T_{\gamma}\left(\left|f_{k}\right|\right)(z)\right|^{2} d V_{\alpha}(z) \leq c_{6}\left[M_{\rho, \gamma}\right]^{2 \sigma} \quad \forall k \geq 1
$$

and, further,

$$
\lim _{k \rightarrow \infty} \int_{\mathbb{B} \backslash S_{\gamma^{1 / 2}}}\left|G_{k}(z)\right|^{2} d V_{\alpha}(z)=0
$$

and (2.11) holds.

Consequently, we have the following estimate on $\left\|\left(C_{\varphi}-C_{\psi}\right)^{*}\left(C_{\varphi}-C_{\psi}\right) f_{k}\right\|$ :

$$
\limsup _{k \rightarrow \infty} \int_{\mathbb{B}}\left|\left(C_{\varphi}-C_{\psi}\right)^{*}\left(C_{\varphi}-C_{\psi}\right) f_{k}\right|^{2} d V_{\alpha}(z) \leq c_{6}\left[M_{\rho, \gamma}\right]^{2 \sigma} .
$$

Letting $\gamma \rightarrow 1^{-}$, we get (2.6) and the compactness of $C_{\varphi}-C_{\psi}$ follows.

Sufficiency. This part is simpler; we follow the method of [6].

By [14], the normalized reproducing kernels of $A_{\alpha}^{2}$ are given by

$$
K_{z}(w)=\frac{\left(1-|z|^{2}\right)^{(n+1+\alpha) / 2}}{(1-\langle w, z\rangle)^{n+1+\alpha}}
$$


and

$$
\int_{\mathbb{B}}\left|C_{\varphi}^{*} K_{z}(w)\right|^{2} d V_{\alpha}(w)=\left(\frac{1-|z|^{2}}{1-|\varphi(z)|^{2}}\right)^{n+1+\alpha} .
$$

Assume that (1.5) fails. To prove that $C_{\varphi}-C_{\psi}$ is not compact, it suffices to show that there exists a sequence $\left(z_{k}\right) \subset \mathbb{B}$, with $\left|z_{k}\right| \rightarrow 1$, such that $\left\|\left(C_{\varphi}^{*}-C_{\psi}^{*}\right) K_{z_{k}}\right\| \nrightarrow 0$. Then $C_{\varphi}^{*}-C_{\psi}^{*}$, and hence $C_{\varphi}-C_{\psi}$ is not compact.

From (1.4) and (2.13),

$$
\left|\left\langle C_{\varphi} K_{z}, C_{\psi} K_{z}\right\rangle\right|^{2}=\left(1-\left|\rho_{\varphi, \psi}(z)\right|^{2}\right)^{n+1+\alpha}\left\|C_{\varphi} K_{z}\right\|^{2}\left\|C_{\psi} K_{z}\right\|^{2} .
$$

We define $\rho_{1}(z):=\left(1-\left|\rho_{\varphi, \psi}(z)\right|^{2}\right)^{(n+1+\alpha) / 2}$ for all $z \in \mathbb{B}$. Then

$$
\begin{aligned}
\left\|\left(C_{\varphi}^{*}-C_{\psi}^{*}\right) K_{z}\right\|^{2} & =\left\|C_{\varphi} K_{z}\right\|^{2}+\left\|C_{\psi} K_{z}\right\|^{2}-2 \operatorname{Re}\left\langle C_{\varphi} K_{z}, C_{\psi} K_{z}\right\rangle \\
& \geq\left\|C_{\varphi} K_{z}\right\|^{2}+\left\|C_{\psi} K_{z}\right\|^{2}-2 \rho_{1}(z)\left\|C_{\varphi} K_{z}\right\|\left\|C_{\psi} K_{z}\right\| \\
& =\left(\left\|C_{\varphi} K_{z}\right\|-\left\|C_{\psi} K_{z}\right\|\right)^{2}+2\left(1-\rho_{1}(z)\right)\left\|C_{\varphi} K_{z}\right\|\left\|C_{\psi} K_{z}\right\| .
\end{aligned}
$$

Since (1.5) does not hold, there exists some sequence $\left(z_{k}\right) \subset \mathbb{B}$ such that $\left|z_{k}\right| \rightarrow 1$ and at least one of $\left(a_{k}\right)$ and $\left(b_{k}\right)$ does not tend to 0 , where

$$
\begin{aligned}
& a_{k}=\rho_{\varphi, \psi}\left(z_{k}\right) \frac{1-\left|z_{k}\right|^{2}}{1-\left|\varphi\left(z_{k}\right)\right|^{2}}=\rho_{\varphi, \psi}\left(z_{k}\right)\left\|C_{\varphi} K_{z_{k}}\right\|^{2 /(n+1+\alpha)} \\
& b_{k}=\rho_{\varphi, \psi}\left(z_{k}\right) \frac{1-\left|z_{k}\right|^{2}}{1-\left|\psi\left(z_{k}\right)\right|^{2}}=\rho_{\varphi, \psi}\left(z_{k}\right)\left\|C_{\psi} K_{z_{k}}\right\|^{2 /(n+1+\alpha)} .
\end{aligned}
$$

By passing to a subsequence if necessary, we may assume that $\lim _{k \rightarrow \infty} a_{k}=a$ and $\lim _{k \rightarrow \infty} b_{k}=b$, and at least one of these limits is nonzero. By symmetry, without loss of generality, we may suppose that $a \neq 0$.

If $a \neq b$, then

$$
\lim _{k \rightarrow \infty}\left(\left\|C_{\varphi} K_{z_{k}}\right\|-\left\|C_{\psi} K_{z_{k}}\right\|\right)^{2} \neq 0 .
$$

If $a=b \neq 0$, we choose a positive $\delta$ such that $\left|\rho_{\varphi, \psi}\left(z_{k}\right)\right|^{2}>\delta$ and

$$
\left(1-\rho_{1}\left(z_{k}\right)\right)\left\|C_{\varphi} K_{z_{k}}\right\|\left\|C_{\psi} K_{z_{k}}\right\|
$$

is bounded away from zero for all $k$. In either case,

$$
\lim _{k \rightarrow \infty}\left\|\left(C_{\varphi}^{*}-C_{\psi}^{*}\right) K_{z_{k}}\right\| \neq 0
$$

which shows that $C_{\varphi}-C_{\psi}$ is not compact. The proof of the theorem is complete.

2.2. Proof of Theorem 1.4. We will make use of Theorem 1.3.

Fix some $\gamma \in[1 / 2,1)$. Consider a bounded sequence $\left(f_{k}\right)$ in $A_{\alpha}^{2}$ that converges to 0 uniformly on every compact subset of $\mathbb{B}$. Our target is to show that

$$
\left\|\left(C_{\varphi}-C_{\varphi_{1}}-\cdots-C_{\varphi_{N}}\right) f_{k}\right\|^{2} \rightarrow 0 \quad \text { as } k \rightarrow \infty .
$$


Define

$$
\begin{gathered}
M_{\varphi, \gamma}:=\min _{i} \sup _{z \in S_{\gamma}<\left[\rho_{i}(z)\right]^{\sigma}<1} \rho_{\varphi, \varphi_{i}}(z) \frac{1-|z|^{2}}{1-|\varphi(z)|^{2}}, \\
M_{\varphi, \rho_{i}, \gamma}:=\sup _{\gamma<\left[\rho_{i}(z)\right]^{\sigma}<1} \rho_{\varphi, \varphi_{i}}(z) \frac{1-|z|^{2}}{1-\left|\varphi_{i}(z)\right|^{2}},
\end{gathered}
$$

and

$$
M_{\varphi, \rho_{i, j}, \gamma}:=\sup _{\gamma<\left[\rho_{i, j}(z)\right]^{\sigma}<1} \rho_{\varphi_{i}, \varphi_{j}}(z)\left(\frac{1-|z|^{2}}{1-\left|\varphi_{i}(z)\right|^{2}}+\frac{1-|z|^{2}}{1-\left|\varphi_{j}(z)\right|^{2}}\right) .
$$

Applying to $\left\|\left(C_{\varphi}-C_{\varphi_{1}}-\cdots-C_{\varphi_{N}}\right) f_{k}\right\|^{2}$ the arguments used to show (2.2)-(2.12), we see that

$$
\begin{aligned}
& \left\|\left(C_{\varphi}-C_{\varphi_{1}}-\cdots-C_{\varphi_{N}}\right) f_{k}\right\|^{2} \\
& \quad \leq\left[M_{\varphi, \gamma}\right]^{2 \sigma}+2 \sum_{j=1}^{N}\left[M_{\varphi, \rho_{i}, \gamma}\right]^{2 \sigma}+2 \sum_{i=1}^{N} \sum_{j=1}^{N}\left[M_{\varphi, \rho_{i, j}, \gamma}\right]^{2 \sigma} .
\end{aligned}
$$

Combining the last inequality and (1.6) yields (2.14).

Thus there exists a compact operator $K$ on $A_{\alpha}^{2}$ such that

$$
C_{\varphi}=C_{\varphi_{1}}+\cdots+C_{\varphi_{N}}+K
$$

which gives (1.7).

REMARK 2.2. As we may observe from the statements of Theorems 1.3 and 1.4, the necessary and sufficient conditions for the compact differences in the case $n=1$ have been generalized to higher dimensions, with the extra assumption that both composition operators $C_{\varphi}, C_{\psi}$ are bounded on the space $A_{\beta}^{q}$ for some $q>0$ and $\beta \in(-1, \alpha)$.

This requirement is not very strict, and when $n=1$, this additional boundedness is automatically satisfied. It is worth considering (some) conditions under which composition operators are bounded in higher dimensions. This will be discussed, for completeness, in the next section.

\section{On the boundedness of composition operators}

In general, for an arbitrary holomorphic mapping $\varphi: \mathbb{B} \rightarrow \mathbb{B}$, the composition operator $C_{\varphi}$ is not bounded on the Hardy space $H^{p}(\mathbb{B})$, nor on the weighted Bergman space $A_{\alpha}^{p}(\mathbb{B})$. A simple counterexample is the following, when $n=2$ :

$$
\varphi\left(z_{1}, z_{2}\right)=\left(2 z_{1} z_{2}, 0\right)
$$

We refer the reader to [1, Section 6.2] for more information on this. 
A characterization of boundedness on Bergman and Hardy spaces is given by the so-called Carleson measure condition, which is usually hard to check. Active research is still going on. Some sufficient conditions for boundedness on Hardy spaces are presented in $[11,12]$. Other sets of necessary and sufficient conditions for boundedness on weighted Bergman spaces have been given in recent papers [2-4]. In these articles, the reader may find further references for recent developments on Bergman spaces.

Since bounded composition operators have an important place in our Theorems 1.3 and 1.4, we now present some significant results about this problems taken from the above-mentioned references.

The first kind of result concerns the domain of definition of a composition operator. Here the question is when $C_{\varphi}$ acts on $A_{\alpha}^{p}(\mathbb{B})$. Define

$$
D_{\xi}=\sum_{k=1}^{n} \xi_{k} \frac{\partial}{\partial \xi_{k}} \quad \text { and } \quad \varphi_{\xi}(z)=\langle\varphi(z) \xi\rangle \quad \forall z \in \mathbb{B}, \xi \in \partial \mathbb{B} .
$$

In [2], the following criterion has been established, extending the result in [11] on Hardy spaces to Bergman spaces.

Theorem 3.1. Let $\varphi: \mathbb{B} \rightarrow \mathbb{B}$ and $\varphi \in C^{3}(\overline{\mathbb{B}})$. For all $\alpha \geq-1, C_{\varphi}$ is a bounded operator on $A_{\alpha}^{p}(\mathbb{B})$ if and only if

$$
D_{\xi} \varphi_{\eta}(\xi)>\left|D_{\tau} D_{\tau} \varphi_{\eta}(\xi)\right|
$$

for all $\xi, \eta, \tau \in \partial \mathbb{B}$ with $\langle\xi, \eta\rangle=0$ and $\varphi(\xi)=\eta \in \partial \mathbb{B}$.

Next, in [3] it was shown that nonvanishing of the Jacobian implies the boundedness of the corresponding composition operators.

THEOREM 3.2. If $\varphi \in \mathcal{O}(\mathbb{B}) \cap C^{2}(\overline{\mathbb{B}})$, then $C_{\varphi}$ is bounded on $A_{\alpha}^{p}(\mathbb{B})$ if $J_{\varphi}(\xi) \neq 0$ for all $\xi \in \partial \mathbb{B}$ for which $\varphi(\xi) \in \partial \mathbb{B}$.

The converse of Theorem 3.2 is false. Indeed, if $\varphi(z)=\left(z_{1}, 0, \ldots, 0\right)$ and $\psi(z)=$ $\left(z_{1}^{2}+z_{2}^{2}, 0, \ldots, 0\right)$, then the Jacobian for both $\varphi$ and $\psi$ are identically zero, while

$$
C_{\varphi}: A_{\alpha}^{p}(\mathbb{B}) \rightarrow A_{\alpha}^{p}(\mathbb{B}) \quad \text { but } C_{\psi}: A_{\alpha}^{p}(\mathbb{B}) \nrightarrow A_{\alpha}^{p}(\mathbb{B}) .
$$

Overall, the boundedness of composition operators in higher dimensions needs further investigation.

\section{Acknowledgements}

The authors thank the referees for the useful remarks and comments that led to the improvement of this paper. Their thanks also go to Jennifer Moorhouse for letting them know about her thesis [6] and to Boo Rim Choe for introducing papers [3, 4] to them. 


\title{
References
}

[1] C. Cowen and B. MacCluer, Composition Operators on Spaces of Analytic Functions (CRC Press, Boca Raton, FL, 1995).

[2] H. Koo and W. Smith, 'Composition operators induced by smooth self-maps of the unit ball in $\mathbb{C}^{n}$, J. Math. Anal. Appl. 329 (2007), 617-633.

[3] H. Koo, M. Stessin and K. Zhu, 'Composition operators on the polydisc induced by smooth symbols', J. Funct. Anal. 254 (2008), 2911-2925.

[4] H. Koo and M. Wang, 'Composition operators induced by smooth self-maps of the real or complex unit balls', J. Funct. Anal. 256 (2009), 2747-2767.

[5] T. L. Kriete and J. Moorhouse, 'Linear relations in the Calkin algebra for composition operators', Trans. Amer. Math. Soc. 359 (2007), 2915-2944.

[6] J. Moorhouse, ' $C^{*}$-algebraic and component structure of composition operators', $\mathrm{PhD}$ Thesis, University of Virginia, 2003.

[7] J. Moorhouse, 'Compact differences of composition operators', J. Funct. Anal. 219 (2005), 70-92.

[8] W. Rudin, Function Theory in the Unit Ball of $\mathbb{C}^{n}$ (Springer, New York, 1980).

[9] J. H. Shapiro, Compositions Operators and Classical Function Theory (Springer, New York, 1993).

[10] J. H. Shapiro and P. D. Taylor, 'Compact, nuclear and Hilbert Schmidt composition operators on $H^{2}$, Indiana Univ. Math. J. 23 (1973), 471-496.

[11] W. R. Wogen, 'The smooth mappings which preserve the Hardy space $H^{2}\left(\mathbb{B}_{n}\right)$ ', Oper. Theory Adv. Appl. 35 (1988), 249-263.

[12] W. R. Wogen, 'On geometric properties of smooth maps which preserve $H^{2}\left(\mathbb{B}_{n}\right)$ ', Michigan Math. J. 54 (2006), 301-306.

[13] K. Zhu, Operator Theory in Function Spaces, 2nd edn (American Mathematical Society, Providence, RI, 2007).

[14] K. Zhu, 'Compact composition operators on Bergman spaces of the unit ball', Houston J. Math. 33 (2007), 273-283.

\author{
XIANG DONG YANG, Department of Mathematics, \\ Kunming University of Science and Technology, 650093 Kunming, \\ Yunnan Province, PR China \\ e-mail: yangsddp@126.com
}

\author{
LE HAI KHOI, Division of Mathematical Sciences, \\ School of Physical and Mathematical Sciences, \\ Nanyang Technological University (NTU), 637371 Singapore \\ e-mail:1hkhoi@ntu.edu.sg
}

